\title{
Prevalence and antibiotic sensitivity of Group B Streptococcus (GBS) infection among antenatal women
}

\author{
Nishita Shettian*, Shetty Theertha Shankar, Manjunath Kamath Ammembal
}

Department of Obstetrics and Gynecology, A. J. Institute of Medical Sciences, Mangalore, Karnataka, India

Received: 04 April 2018

Accepted: 09 April 2018

*Correspondence:

Dr. Nishita Shettian,

E-mail: drnishitafernandes@yahoo.com

Copyright: (C) the author(s), publisher and licensee Medip Academy. This is an open-access article distributed under the terms of the Creative Commons Attribution Non-Commercial License, which permits unrestricted non-commercial use, distribution, and reproduction in any medium, provided the original work is properly cited.

\begin{abstract}
Background: Group B Streptococcus (GBS) infection in pregnant women is a major concern as it causes septicemia in neonates which is fatal and potentially life threatening. Objectives of present study were to study the distribution of Group B streptococcus infection among pregnant women at term, to understand if penicillin is still effective as the drug of choice, to determine the alternate drug of choice in women with Group B streptococcus infection if resistant to penicillin and analyze the proportion of Group B Streptococcus infection as a vaginal commensal.

Methods: This is a hospital based prospective study comprising of 350 pregnant women at term gestation. Vaginal swabs as well as neonatal nasal swabs following delivery were collected under aseptic precautions and sent for culture and sensitivity.

Results: Vaginal swab culture reports of 308 women out of 350 showed a growth. Candida albicans (23.8\%), Enterococcus (5.6\%), Escherichia coli (4.2\%), MRSA (4.2\%), Staphylococcus aureus (1.4\%) and Group B Streptococcus $(1.4 \%)$ were the organisms isolated. Penicillin is still the drug of choice for group B Streptococcal infection in antenatal women with Linezolid being the alternate drug of choice. None of the neonatal nasal swabs revealed any growth on culture and sensitivity.

Conclusions: This study does not recommend routine screening for Group B Streptococcus in all pregnant women unless they have a positive history in previous pregnancies. Our study also revealed various other organisms which could be responsible for increasing the risk of maternal and neonatal morbidity.
\end{abstract}

Keywords: Commensal, Culture, Group B Streptococcus, Penicillin

\section{INTRODUCTION}

Group B Streptococcus infection in pregnant women is a fatal and life-threatening condition as it has a tendency to cause septicemia in neonates. ${ }^{1}$ It is alarming to know that GBS infection is linked with an increased risk of chorioamnionitis as well as neonatal septicemia. ${ }^{2,3}$

GBS is a Gram positive bacteria also known as Streptococcus agalactiae. They are often found in the vagina, rectum, urinary bladder and intestines and isolated from body fluids like CSF, blood, urine and vaginal secretions. They tend to be asymptomatic but can potentially be lethal. $15-45 \%$ of all pregnant women are believed to be colonized with GBS and $20-40 \%$ of these are colonized during labour. In 1-2\% of the pregnant women infected with GBS, the infection can be passed on to the fetus and this can prove to be fatal. Though rare, the neonate usually acquires GBS infection from the mother during labour. Most infants will present with illness shortly after birth and $90 \%$ have been found to show clinical signs and symptoms within 24 hours of life. ${ }^{4,5}$ To deal with this situation, a routine screening for GBS may be offered to all pregnant women at term gestation. ${ }^{6}$ The likelihood of subsequent pregnancies being affected is $38 \%$ in women with a history of GBS 
infection in a previous pregnancy or has previously given birth to a GBS infection positive baby. ${ }^{7}$ Hence these women may be screened at term for GBS infection based on their past history.

Studies done in the United Kingdom have stated that the incidence of GBS as a vaginal commensal is $21 \%$ worldwide. $^{8}$ The prevalence of GBS was found to be $2.3 \%$ based on a prospective study done in South India. ${ }^{9}$ However, there are very few studies done in our country regarding the prevalence of GBS in India. As a result there is no sufficient data to address this deficit regarding the prevalence and outcomes of Group B Streptococcus infection in our country.

According to the National Screening Committee of the United Kingdom, unless proven that antenatal screening for GBS infection is beneficial, routine screening is not recommended. ${ }^{10}$ The use of antibiotics in most units in our country is much more empirical and without a definite protocol. Penicillin is the antibiotic of choice currently being used for the treatment of GBS infection but there is no data suggesting if the strain has developed resistance to penicillin.

Present study aims at assessing the incidence of GBS infection in our population and to determine the antibiotic sensitivity for Group B Streptococcus infection by comparing it to the available data to find out the appropriate drug of choice. The purpose of this study is to also justify the need for universal screening of all pregnant women at term for GBS infection and to study the outcomes associated with it.

Objectives of present study were to study the distribution of Group B streptococcus infection among pregnant women at term, to understand if penicillin is still effective as the drug of choice, to determine the alternate drug of choice in women with Group B streptococcus infection if resistant to penicillin and analyze the proportion of Group B Streptococcus infection as a vaginal commensal.

\section{METHODS}

This is a hospital based prospective study comprising of pregnant women at term gestation visiting the out patient department of Obstetrics and Gynecology. A sample size of 350 antenatal women were recruited in the study.

\section{Inclusion criteria}

- Pregnant women,

- Term Gestation (37-42 weeks)

- No medical complications.

\section{Exclusion criteria}

- Preterm labour,

- women with urinary complaints,

- women already on antibiotics,
- women who do not give consent.

A detailed history and examination of these pregnant women was done and a written informed consent was taken from those willing to be a part of the study after explaining the procedure to them in detail.

Vaginal swabs were then collected from these antenatal women at term gestation in the Out Patient Department under aseptic precautions ensuring the patient's privacy at all times and in the presence of a chaperone. The vaginal swabs were then sent for culture and sensitivity to the central microbiology laboratory. These women were followed up on a regular basis and after delivery neonatal nasal swabs were subsequently taken and sent for culture and sensitivity.

The Culture and sensitivity reports were then collected and compiled. The women testing positive for Group B Streptococcus infection were treated with the appropriate antibiotic based on the antibiotic sensitivity report. Statistical analysis of the data was done using Chi square test and a $\mathrm{P}$ value of $<0.05$ was considered statistically significant.

\section{RESULTS}

A total of 350 pregnant women at term gestation were enrolled in the study. Vaginal swab culture and sensitivity reports of 308 women out of 350 i.e. $88 \%$ of the pregnant women recruited in our study revealed a growth on culture and sensitivity (Figure 1).

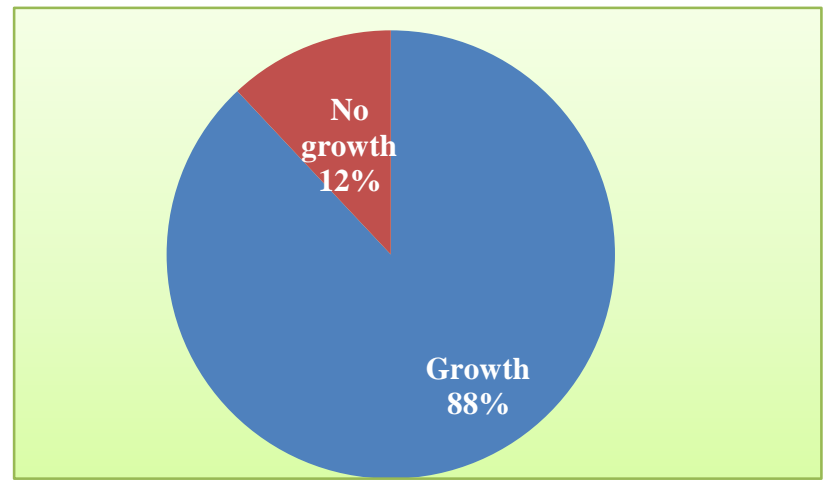

Figure 1: Distribution of growth on culture and sensitivity.

$126(36 \%)$ women were primiparous while $224(64 \%)$ women were multiparous among those who showed a growth. Distribution of women as per age revealed that $27.7 \%$ belonged to 20-25 years, majority of women $(45.7 \%)$ were in the age group of $25-30$ years, $25.1 \%$ were $30-35$ years and only $1.4 \%$ belonged to the age group of 35-40 years.

The following organisms were isolated from the vaginal swab culture (Figure 2). 


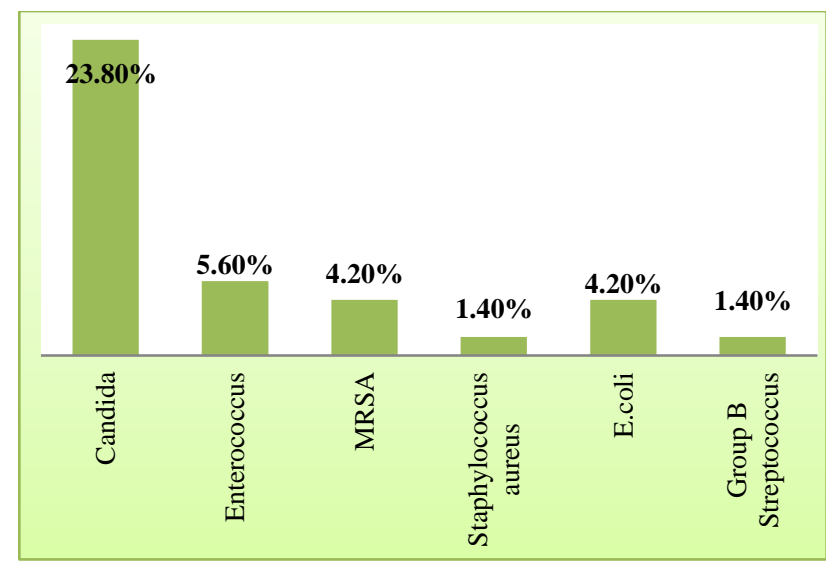

Majority of the organisms isolated was found to be Candida albicans whereas Group B streptococcus turned out to be the least isolated organism thus implying that the prevalence of GBS in our population is still very low. In contrast to our study the preponderance of other organisms isolated could also be a potential cause of neonatal infection and other maternal complications like preterm labour and chorioamnionitis. This would require more number of studies on these organisms and their effects on pregnant women in order to prove the same.

Penicillin is still found to be the drug of choice for group B Streptococcal infection in antenatal women showing $100 \%$ sensitivity rate (Table 1).

Figure 2: Distribution of organisms.

Table 1: Percentage of antibiotic sensitivity of the isolated organisms.

\begin{tabular}{|c|c|c|c|c|c|}
\hline \multirow[t]{2}{*}{ Antibiotic } & \multicolumn{5}{|l|}{ Organisms } \\
\hline & Enterococcus & E. coli & Group B Streptococcus & MRSA & Staphylococcus aureus \\
\hline Cefotaxime & - & 87 & - & 37.5 & 47.1 \\
\hline Linezolid & 100 & - & 100 & 100 & 100 \\
\hline Cefuroxime & - & 71 & -4 & 18 & 67 \\
\hline Clindamycin & - & - & - & 70 & 70.6 \\
\hline Gentamicin & 90 & 77.3 & 80 & - & - \\
\hline Amoxyclav & 70 & 47 & 80 & 25 & 25 \\
\hline Ampicillin & 33 & - & 80 & - & - \\
\hline Cotrimoxazole & - & - & - & 20 & 20 \\
\hline Penicillin & 70 & - & 100 & - & 10 \\
\hline Azithromycin & - & - & - & 55.5 & 55.5 \\
\hline Ceftriaxone & - & 75 & - & 25 & 47 \\
\hline
\end{tabular}

All strains of MRSA were found to be $100 \%$ sensitive to Linezolid. Clindamycin $(70.6 \%)$ and Azithromycin $(55.5 \%)$ also had high sensitivity rates. All the other bacterial isolates like Enterococcus, E. coli, Staphylococcus aureus including Group B streptococcus were all found to have $100 \%$ sensitivity to Linezolid. The women in whom Group B Streptococcus was detected, all the isolates were found to be sensitive to penicillin and hence the same was used to treat these women. In those resistant to penicillin our study found that Linezolid also had a $100 \%$ sensitivity rate and hence could be chosen as an alternate drug of choice to treat GBS infection.

However, none of the neonatal nasal swabs revealed any growth on culture and sensitivity including those detected to have Group B Streptococcus infection which highlights the fact that they were effectively treated with the appropriate antibiotic of choice.

\section{DISCUSSION}

Majority of the participants in present study revealed a growth on culture and sensitivity of vaginal swabs (88\%).
This is in contrast to the study conducted by Wood et al where only $8 \%$ of the women confirmed the presence of bacteriuria. $^{3}$

According to a study by Sharmila et al 300 women were enrolled in the study. ${ }^{9}$ Group B Streptococcus was isolated from 7 out of 300 patients which accounted to a $2.33 \%$ colonization rate whereas present study showed a colonization rate of only $1.4 \%$.

In the study done by Wood et al, 14 women were isolated with Group B Streptococcal infection. ${ }^{3}$ The only organism that exceeded the number of GBS positive women was Escherichia coli.

In this study, majority of the women were positive for Candida infection followed by Enterococcus. GBS was the least isolated organism. This revealed the fact that GBS infection was not so common among our population.

Majority of women who showed a growth in the culture and sensitivity reports were multiparous. This was in 
accordance to a study carried out in West Indies by Orrett et al where colonization rates were found to be higher among multiparous women than primiparous. ${ }^{11}$

$45.7 \%$ of women were in the age group of $25-30$ years and $27.7 \%$ were in the age group of $20-25$ years. The age distribution of the participants was found to be similar to the study by Sharmila et al where age of the participants ranged from 18 years to 39 years with a mean \pm SD of $23.28 \pm 2.95$ years.

$25.1 \%$ patients were in the age group of $30-35$ years and $1.4 \%$ were in the age group of $35-40$ years. ${ }^{9}$ According to a study by Orrett et al, Group B streptococci were isolated more frequently from women $>24$ years of age $(36.6 \%)$ than those younger than 24 years $(26.9 \%)$ which was in accordance to present study. ${ }^{11}$

In the patients where Group B Streptococcus was detected, Pencillin was the antibiotic of choice used to treat the same and all the isolates were sensitive to penicillin which was in accordance to a study done by Sharmila et al. ${ }^{9}$

All strains of MRSA were found to be $100 \%$ sensitive to Linezolid. Higher sensitivity rates were found with Clindamycin and Azithromycin. Among the Enterococcus species, highest sensitivity was found with Linezolid (100\%) followed by Gentamycin (90\%), Amoxyclav (70\%) and Penicillin (70\%)

Of the total number of swabs reviewed, Candida was the predominant organism isolated in our study, while bacterial growth identified were predominantly Enterococcus followed by MRSA and E. coli.

The least isolated organisms were Group B Streptococcus and Staphylococcus aureus. This was in accordance to a study by Orish VN et al where Candida species were the most prevalent organisms isolated in the study. However, it was in contrast to the finding that Escherichia coli was the predominant aerobe followed by Staphylococcus aureus as observed by Verner Ndudiri Orish.$^{12}$

Nasal swabs were obtained from all the neonates following delivery. However, none of the nasal swabs revealed any growth on culture and sensitivity.

\section{CONCLUSION}

Majority of the culture and sensitivity reports did not reveal Group B Streptococcus infection. Hence present study does not recommend routine screening for Group B Streptococcus in all pregnant women.

However, women with a history of Group B streptococcus infection in a previous pregnancy or those who have given birth to a Group B Streptococcus infection positive baby in the past require screening in the antenatal period. A number of other organisms were isolated which reflects the need to conduct further studies to know the distribution of these organisms among antenatal women and their effects on pregnancy.

Thus, present study suggests the need to screen for other organisms which might be responsible for increasing the maternal and neonatal morbidity but in order to substantiate our results more studies on a larger scale are warranted.

\section{Funding: No funding sources}

Conflict of interest: None declared

Ethical approval: The study was approved by the Institutional Ethics Committee

\section{REFERENCES}

1. Kenyon S, Brocklehurst P, Blackburn A, Taylor DJ. Antenatal screening and intrapartum management of Group B Streptococcus in the UK. BJOG. 2004;111:226-30

2. Anderson BL, Simhan HN, Simons KM, Wisenfeld HC. Untreated asymptomatic group B streptococcal bacteriuria early in pregnancy and chorioamnionitis at delivery. Am J Obstet Gynecol. 2007;196:524.e15 .

3. Wood EG, Dillon HC Jr. A prospective study of group B streptococcal bacteriuria in pregnancy. Am J Obstet Gynecol. 1981;140:515-20.

4. Heath PT, Balfour GE, Tighe H, Verlander NQ, Lamagni TL, Efstratiou A, HPA GBS Working Group. Group B streptococcal disease in infants: A case control study. Arch Dis Child. 2009;94:674-80.

5. Vergnano S, Menson E, Embleton N, Russell AB, Collinson A, Heath P. Missed opportunities for preventing group B streptococcus infections. Arch Dis Child Fetal Neonatal Ed. 2010;95:F72-3.

6. Prevention of perinatal group B streptococcal disease: A public health perspective. Centers for Disease Control and Prevention. MMWR. 1996;45:1-24.

7. Shah V, Ohlsson A; Canadian Task Force on Preventive Health Care. Prevention of Early-onset Group B Streptococcal (GBS) Infection in the Newborn: Systematic Review and Recommendations. CTFPHC Technical Report \#016. London, ON, Canada: Canadian Task Force; 2001.

8. Coulborn T, Gilbert R. An overview of the natural history of early onset group B streptococcal disease in the UK. Early Hum Dev. 2007;83:149-56.

9. Sharmila V, Joseph NM, Babu TA, Chaturvedula L, Sistla S. Genital tract group B streptococcal colonization in pregnant women: A South Indian perspective. J Infect Dev Ctries. 2011;5(8):592-5.

10. UK National Screening Committee. Group B Streptococcus: The UK NSC policy on Group B Streptococcus screening in pregnancy, London: NSC; 2008 Avaliable at http://www.screening.nhs.uk/groupbstreptococcus 
11. Orrett FA. Colonization with Group B Streptococci in pregnancy and outcome of infected neonates in Trinidad. Pediatr. Int. 2003 Jun;45(3):319-23.

12. Orish VN, Ofori-Amoah J, François M, Silverius BK, Mensah EK. Microbial and antibiotic sensitivity pattern of high vaginal swab culture results in sekondi-takoradi metropolis of the western region of ghana: retrospective study. Eur J Clin Biomed Sci. 2016;2(5):45-50.

Cite this article as: Shettian N, Shankar ST, Ammembal MK. Prevalence and antibiotic sensitivity of Group B Streptococcus (GBS) infection among antenatal women. Int J Reprod Contracept Obstet Gynecol 2018;7:1768-72. 